\title{
Reactions of carboline
}

\author{
Christophe Dardonville, Henri Virelizier, Jean Boivin and Christopher K. Jankowski* \\ CE-Saclay, DPE/SPEA/SAIS, bat. 391, 91191 Gif-sur-Yvette, France
}

Received 13 July 1996

Accepted 11 March 1997

\begin{abstract}
The reactions of carboline under various oxidative conditions are reported. The soft aerobic radiolysis, hydroxylation and rearrangement transformations are studied using isotopic labelling and GC-MS techniques.
\end{abstract}

Keywords: Soft radiolysis of indolic alkaloid, oxidations of indolic alkaloid

\section{Introduction}

The oxidative transformation of indole alkaloids, belonging to the tetrahydro-beta-carboline (1) family, under various biological, biomimetical or chemical conditions was extensively studied by our group [1-6]. The parent compound 1 easily underwent a series of monohydroxylation with Cytochrom P 450 [6], as well as with Gif [1-4] or FeII/FeIII red-ox systems [5,6]. Although the yields of hydroxylation are generally low, numerous interesting side products, e.g., keto derivatives, diols, etc., are obtained. In all reactions studied an important level of autooxidation of alkaloids as well as several coupling reactions were observed. In this study we report a soft gamma radiolysis of $\underline{1}$ under different aerobic conditions, its Barton's photolysis in presence of N-hydroxypyridone-2-thione (2) [7-9], together with its lead tetraacetate oxidation. This last reaction, proceeding via the oxidation of 7 a carbon of indole alkaloids to the 0 -acetate ( $\underline{3})$, lead through a rearrangment of the skeleton to an oxindole lactam $\underline{4 b}$ or according to Finch scheme [10] to the indolinone ( $\underline{4 a})$. Both reactions should be considered as possible under the biomimetic oxidation of 1 framework.

The application of radiolysis to alkaloids, or to the natural products in general, is a very appealing way to achieve their transformation. However, although unusual, the radiolysis is a highly unspecific process which can enhance several unsuitable and unexpected reactions as well as it can lead to an extremly complex post-reaction mixtures of products. The behavior of natural products under soft radiolysis remains a fascinated and relatively unexplored field.

All reactions presented here were carefully monitored by the GC-MS technique, the identification of tetrahydro-beta-carboline derivatives were confirmed by comparison with available compounds coming from our previous synthesis and by selected deuterium labelling experiments.

\footnotetext{
${ }^{*}$ Corresponding author. Address: FESR, Université de Moncton, Moncton, NB, Canada, E1A 3E9.
} 


\section{Results and discussion}

Tetrahydrocarboline (1a) irradiated with a visible light in the presence of $\underline{2}$ in aromatic solvent under aerobic conditions (benzene or chlorobenzene) produce, as expected, three disulphides issuing from the dispropotionation of intermediate sulphide radicals ( $\underline{8 \mathrm{a}}, \underline{8 \mathrm{~b}}$ and $\underline{8 \mathrm{c}}$; total yield of $18.7 \%)$ [8,9], as well as two unseparated C-4 and C-6 substituted thiopyridylcarbolines $(\underline{7 d})(m / z 335)$ obtained in a $2: 1$ ratio, with a total yield of $1.8 \%$. The absence of higher yield of these compounds and of three hydroxycarbolines $(\underline{7}, 0.8 \%)$ under these conditions could be explained by the small yield of $\mathrm{OH}$. radical and by the fact that these radicals could abstract hydrogen from the methylenes especially when these are activated by heteroatom and then allow the carboline-S-thiopyridyl coupling reactions.

It is worthy to note that the abstraction of labile hydrogen atom by the $\mathrm{OH}$. generated by cleavage of $\underline{2}$ is much more efficient when the substrate is at the same time the reaction solvent $[8,9]$. These conditions cannot be acheived when a high molecular weight substrates are used, as it was in our case (Figs 1 and 2).

When the same reaction, under visible light, is performed under anaerobic conditions (under $\mathrm{N}_{2}$ atmosphere) in a presence of $\underline{2}$, the carboline $\underline{1 a}$ underwent the same kind of transformation. The

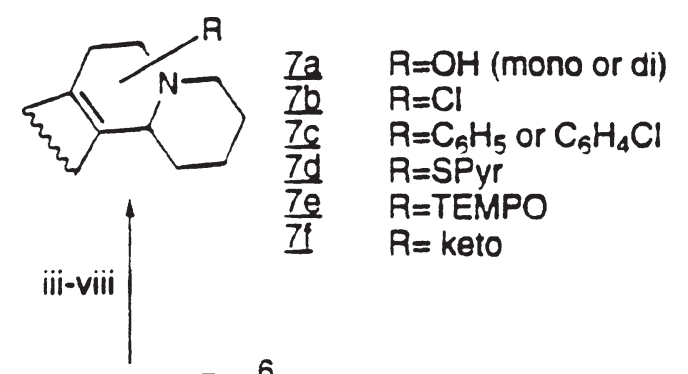

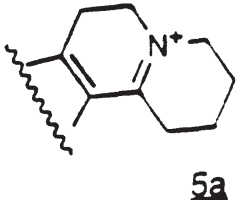

$\underline{5 a}$<smiles>[Y]C1C2=Nc3ccccc3C2(O)CCN1C</smiles>

3a $\quad R=A c$

$\frac{3 a}{3 b} \quad R=H$

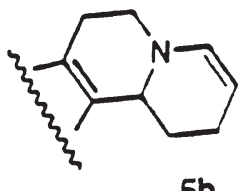

$5 \mathbf{b}$

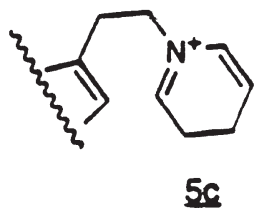

Intermediates<smiles>On1ccccc1=S</smiles>

2

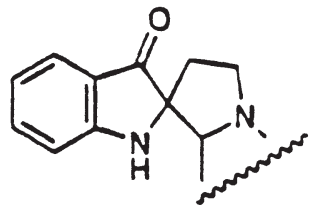

$4 a$<smiles>CC1CCCC12C(=O)Nc1ccccc12</smiles>

46

Fig. 1. (i) $\mathrm{MeO}^{-}$, (ii) $\mathrm{H}^{+}$, (iii) chlorobenzene, radiolysis, (iv) chlorobenzene, radiolysis, N-hydroxythiopyridone, (v) N-hydroxy thiopyridone, sunlight or tungsten lamp, dichloromethane or pyridine, (vi) TEMPO, dichloromethane, (vii) cumyiperoxide, dichloromethane, (viii) $\mathrm{Co}(\mathrm{AcAc})_{3}$, toluene, oxygen. 
product profile remains the same with a reduction, by half, of hydroxylation products yield. This reduction could be related to two reasons, the autooxidation of indole alkaloid as well as to the influence of atmospheric dioxygen on the oxidation reaction level. Although temptating, the difference in the isolated product yields under aerobic-anaerobic condition shift cannot be unambiguously, because of a very small yield of the $\underline{7 a}$ products, related to the atmospheric oxygen presence.

The aerobic radiolysis of $1 \mathrm{a}$ under Co bomb gamma rays $(150 \mathrm{~min})$ conditions in chlorobenzene was then performed with and without presence of $\underline{2}$. The radiolysis of $\underline{1}$ in chlorobenzene alone mainly lead to formation of three major compounds chlorodiphenyl, dichlorodiphenyl and diphenyl, $\underline{6 a}-\underline{6 c}$, respectively, as well as to smaller quantities of the dibenzene dimer $(\underline{6 \mathrm{~d}})$ and to a small quantity of hydroxycarboline $(\underline{7 a})$, chlorophenylcarboline $(\underline{7 \mathrm{c}})$, dihydroxycarboline $(\underline{7 \mathrm{a}})$ and phenylcarboline $(\underline{7 \mathrm{c}})$, respectively, with following yields: $2.7,1.9,3.1$ and $2.3 \%$, expressed as a percentage of transformation of the substrate $1 \mathrm{a}$. The position of substitution of these radicals on the carboline ring was not rigorously established, however the usually activated position of alpha to $\mathrm{N}_{5}$ and benzylic carbon $\mathrm{C}_{7}$ are the preferred target of these reactions.

When this radiolysis is performed in the presence of $\underline{2}$ three groups of products were obtained. The two most abundant groups corresponded to chlorobenzene radiolysis products (ca. $45 \%$ of the mixture of $\underline{6 a}-6 \mathrm{~d}$ compounds mentioned above; yields of $2.3,27.0,2.1$ and 13.7\%); and to three thiopyridone originated dimers $(\underline{8 a}-\underline{8 c})$ in the yields range of $40 \%$ of transformation of compond 2 and the ratio of $3: 2: 2$ of compounds $\underline{8 \mathrm{a}}: \underline{8 \mathrm{~b}}: \underline{8 \mathrm{c}}$. The last group of products containing the carboline moiety showed in order of decreasing abundance: S-2-pyridyloxycarboline (3.1\%), chlorophenylcarboline $(2.6 \%)$,

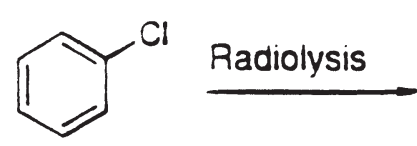<smiles>c1ccc(-c2ccccc2)cc1</smiles>

$6 a$<smiles>Clc1ccc(-c2ccccc2)cc1</smiles>

$\underline{6 b}$<smiles>Clc1cccc(-c2ccccc2Cl)c1</smiles>

$\underline{6 c}$<smiles>c1ccc2c(c1)-c1ccccc1-2</smiles>

$6 d ?$

Radicals formed:<smiles>[Si]c1ccccn1</smiles>

$\mathrm{HO}^{\circ}$

$\mathrm{Cl}^{\circ}$<smiles>[c]1ccccc1</smiles><smiles>CCC1CCCCC12CCc1c2[nH]c2ccccc12</smiles>

\section{Dimers from 2:}<smiles>Sc1ccccn1</smiles>

89<smiles></smiles>

$8 b$<smiles>O=S(c1ccccn1)c1ccccn1</smiles>

$8 \mathrm{c}$

Fig. 2. 
S-2-pyridylcarboline (1.9\%), hydroxycarbolines (1.7\%) and traces of dihydroxy (1.1\%), chloro $(0.9 \%)$ and phenyl $(0.7 \%)$ carbolines. Comparing these last two oxidations the addition of $\underline{2}$ has increased the relative yield of hydroxycarbolines (7a) by a factor of two and in general has activated the carboline coupling reaction. On the other hand the addition of thiols to methylenes seems to be catalysed by the dioxygen, because under anaerobic conditions the autooxidation of indole alkaloids under radiolysis was slightly lowered [12-15].

Although the structure of isomeric hydroxy carbolines $\underline{7 \mathrm{a}}$ and S-pyridylcarbolines $\underline{7 \mathrm{~d}}$ was not precisely established, it seems, from the isotopic labelling experiments and from the fragmentation of these compounds under mass spectrometry electronic impact conditions, that the S-pyridyl radicals are placed on the alicyclic part of the carboline $\underline{1 \mathrm{a}}$ alpha to nitrogen $\mathrm{N}_{5}$ position, and that the major hydroxylation takes place on the $\mathrm{C}_{6}$ of the 1 as well. At this moment there is no evidence for the addition of the ortho thiopyridyl radical to the pyrrolic double bond of the indole cycle.

The radiolysis of $\underline{2}$ alone in chlorobenzene leads to obtaining seven identified products, with a total yield of $7 \%$, which were in order of decreasing abundance dithiopyridine dimer $(1.9 \%)$ followed by S-phenyl 2-thiopyridine (1.8\%), chlorodiphenyl (1.3\%), chlorohydroxydiphenyl (0.6\%), bisdiphenylether $(0.45 \%)$, dichlorodiphenyl $(0.4 \%)$ and diphenyl $(0.1 \%)$ as well as six unidentified minor reaction products.

The radiolysis described here has certainly improved the yield of the oxidation products of this alkaloid, probably due to the hydroxylation via the $\mathrm{OH} \cdot$ radical. However, the necessary use of high boiling point solvent (chlorobenzene) induced several secondary reactions of this fragile molecule. Radiolysis remains a very uncontrolled and chaotic reaction and further optimisation of reaction conditions is difficult because of a still limited access to the experimental reactors, a little say in choosing the experimental conditions and the generally speaking high cost of these experiments. Despite these factors and because of the important biological role of indolic compounds, the study of the behavior of this moiety under radiolysis is both interesting and necessary.

Most popular chemical oxidants are readily oxidizing the indole ring. In order to complete this study, the search for rearrangement reaction products from 1 under different oxidative conditions was performed. These products could be present in many biomimetic oxidation reactions, however, they have never been detected yet in post-oxidative mixtures. Among the others, the fragility of the indolic aromatic ring under oxidation justified this rearrangement. The lead tetraacetate oxidation of carboline 1a, according to the Finch procedure $[10,11,16,17]$ developed for more substituted indole alkaloids is leading to expected beta-acetoxyindoleine (3a). This compound when treated in mild acidic or basic (e.g., sodium methoxide) conditions should rearrange to oxindole lactam ( $\underline{4 b})$ or to the indolinone ( $\underline{4 \mathrm{a}})$ skeleton systems. The rearrangement of $\underline{3 \mathrm{a}}$ under basic conditions led to obtaining the oxidised product at $m / z 242$ which was identified as indolinone $\underline{4 a}(m / z 130$, 96, etc.).

The rearrengement of $\underline{3 a}$ under acidic conditions led to obtaining two products, one being deprotected, beta hydroxy carboline $\underline{3 \mathrm{~b}}$, the second one underwent the rearrangement to the oxindole displaying the lactam bond $(m / z 241 \mathrm{M}-\mathrm{H}, 149,97$, etc.). When the crude mixture of the lead tetraacetate oxidation of $\underline{1 \mathrm{a}}$ is treated with $\mathrm{NaBD}_{4}$ according to the Gribble procedure [18-20] the intriguing isomeric compound is obtained. This compound is displaying characteristic $\mathrm{M}-\mathrm{D}^{+}$ion at $m / z 225(100 \%)$. During the carboline 1 synthesis, when reaction mixture is treated with $\mathrm{NaBD}_{4}$ instead of $\mathrm{NaBH}_{4}$, the carboline is monodeuterated in position $4\left(\mathrm{~m} / z 227 \mathrm{M}^{+}\right)$, displaying the $100 \%$ ion at $m / z$ 226. The same reaction carried out in deuterated methanol $\left(\mathrm{CH}_{3} \mathrm{OD}\right)$ is producing a triple deuterated product (on the indolic $\mathrm{N}, \mathrm{C}_{3}$ and $\mathrm{C}_{4}$ ) which is displaying a $100 \%$ ion at $\mathrm{m} / z 228$. One of these deuterium atoms $(\mathrm{N}-\mathrm{D})$ is easily reexchanged with an addition of the methanol. 
In order to investigate the intermediates of the lead tetraacetate oxidation of $\underline{1 a}$ the reduction of the intermediate $\underline{3 a}$, with either sodium borohydride or lithium aluminium hydride was performed. When this compound is reacted with lithium aluminium deuteride the monodeuterated $12 \mathrm{~b}-\mathrm{d}_{1}$ carboline $\underline{1 \mathrm{a}}$ formation is observed. This result means that the lead tetraacetate oxidation of $1 \mathrm{a}$ could proceed via the immonium $5 \mathrm{a}$ intermediate as well. In a similar manner the presence of the intermediate $\underline{5 b}$ was confirmed $\left(\mathrm{M}^{+}\right.$ion at 224 , didehydrogenated carboline, followed by an $\mathrm{M}-\mathrm{H}^{+}$ion at 223) during the carboline synthesis. The addition of a $\mathrm{CH}_{3} \mathrm{O}$-ion to the imine $\mathrm{N}_{5}-\mathrm{C}_{12 \mathrm{a}}$ intermediate was not observed. The results obtained from deuteration experiments on both intermediates ( $\underline{5}$ and $\underline{6})$ enabled to better characterise the enamine $\mathrm{N}_{5}-\mathrm{C}_{4}=\mathrm{C}_{3}$, azadiene and imine $\mathrm{N}_{5}-\mathrm{C}_{12 \mathrm{a}}$ systems present in the $\mathrm{C} / \mathrm{D}$ rings junction proximity of this tetracyclic alkaloid according to the intermediates discussed by Barton [7, 17] and postulated by Gribble [18-20]. It is finally interesting to notice that both rearranged carboline derivatives, both bearing the spiro $\mathrm{C}$ ring,were not detected in various biomimetic (e.g., Gif IV, GifOrsay, Fenton, picolinate FeII) as well as in the cytochrome P450 and the radiolytic oxidations of $\underline{1 \mathrm{a}}$ reported in this paper $[5,6]$.

In order to evaluate the radical formation capacity of several methylenes of the carboline the alkaloid was reacted with TEMPO. This reagent, as the natural source of the radicals, can perform the hydrogen abstraction as well as when used in excess, which was the case, it can form the 2,2,6,6-tetramethyl-1piperidinyloxy substituted products and eventually under aerobic conditions the oxidised compounds as well. The use of TEMPO is sometimes performed in order to evaluate the capacity of specific substrate molecule to give the radicals via the hydrogen abstraction and to mark the most active methylenes [21]. When the carboline 1a is treated with TEMPO in methylene chloride solution and worked up with water the formation of approximately $0.01 \%$ of hydroxycarbolines $\underline{7 a}$ trace quantities were observed.

One more attempt was performed to form the hydroxy carbolines and oxidise chemically this alkaloid. The CoIII-acetylacetonate oxidation of 1 in toluene and constant oxygen flow for $12 \mathrm{~h}$ is leading to several products. In this reaction, for instance [22], the allylic hydroxylation and allylic ketonisation of the cyclohexene system together with four vinylic compounds formation was observed: the epoxidation and the rearrangement of this epoxide to the ketone which in the case of carboline (1) should lead to either aminoketone or spiro lactame rearranged products. The last expected product in this reaction is the simple hydroxylation on either of two vinylic carbons of the alpha, beta indolic double bond usually absent in the indolic oxidation, except in the lead tetraacetate oxidation previously described in this paper. The clear identification of hydroxycarbolines $(\underline{7 a})$, representing $17 \%$ of transformed starting material together with benzyl alcohol originated from the allylic hydroxylation of the solvent (toluene) as well as a small quantity of the ketocarboline (7f) was confirmed. However, the cobalt III acetonylacetate catalysed oxidation of the model cyclohexene has led respectively to a major fraction corresponding to the allylic ketonsation (90\%) followed by cyclohexanone, hydroxycyclohexene and 1,2-epoxycyclohexene (total yield of these three compounds 7\%) and some cyclohexanol and cyclopentyl carboxylic acid (3\%). In this respect, our results, when combined to an overhelming quantity of benzyl alcohol somewhat overlooked by the Mexican report, are giving a more complete picture of this reaction [22,23] analysed with more rigorous control by the GC-MS technique (Fig. 3).

The last attempted experiment was the cumene hydroperoxide oxidation of $\underline{1 \mathrm{a}}$ in a methylene chloride solution, leading to traces of hydroxylation of the alkaloid, probably due to the autooxidation and the absence of both cumenoxyl-alkaloid coupling products or the addition of cumenoxyl radical to the indolic double bond expected. 


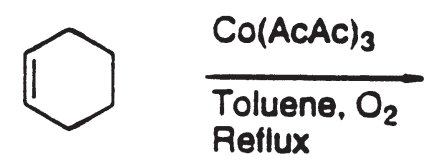<smiles>OCc1ccccc1</smiles><smiles>OC1C=CCCC1</smiles><smiles>O=C1CCCCC1</smiles><smiles>O=C1C=CCCC1</smiles><smiles>C1CCC2OC2C1</smiles>

Fig. 3. Model reaction.

\section{Experimental}

All products and reagents were purchased from Coger Company; most of them originated from Aldrich Chemicals or affiliated suppliers.

\subsection{GC-MS identification of reaction mixtures}

The gas chromatography-mass spectrometric analysis of reaction mixtures for most of the reaction involving the alkaloids and the model oxidation reactions were carried out on a Nermag R-10-10 mass spectrometer (electron impact, positive ions, $70 \mathrm{eV}$ ) coupled to a Delsi DC 700 gas chromatograph using CP SIL 50m ID 0.3u column and the appropriate temperature programming.

\subsection{Photolysis of $\underline{1 a}$ in presence of $\underline{2}$}

The reaction was carried out as $10 \%$ chlorobenzene solution of the alkaloid using the UV Hanovia $1 \mathrm{~L}$ photochemical reactor lamp, $100 \mathrm{~W}$ for $10 \mathrm{~h}$ or sunlight for two months. The ratio of $\underline{1 a}$ to $\underline{2}$ products was ca. $3: 1$. The whole reaction mixture was analysed by GC-MS. The overall reaction yield referred to the starting alkaloid conversion was 17 and $9 \%$, respectively.

The same reaction carried out in benzene or in methylene chloride led to the same product profile.

\subsection{Anaerobic photolysis of $\underline{1 a}$ in the presence of $\underline{2}$}

The photolysis of $\underline{1 \mathrm{a}}$ under $\mathrm{N}_{2}$ atmosphere was carried out as in the previous case using the UV reactor for $10 \mathrm{~h}$. The reaction mixture was analysed by the GC-MS (11\% of conversion of the alkaloid).

\subsection{Radiolysis}

The $10 \%$ solution of carboline $1 \mathrm{a}$ in chlorobenzene was radiolysed under aerobic conditions with Co 60 bomb (Cigal, CEA) for 150 min using $1.8 \mathrm{kGy} \mathrm{h}^{-1}$. The mixture was cooled down and analysed by GC-MS.

(A) Radiolysis of pure chlorobenzene witness sample yielded the mixture of four compounds: diphenyl (2.3\%), chlorodiphenyl (27.1\%), dichlorodiphenyl (2.1\%) and dibenzene dimer $(13.7 \%)$. All yields are referred to conversion of starting material. 
(B) Radiolysis of ca $10 \%$ solution of $1 \mathrm{a}$ in chlorobenzene resulted in ca $23 \%$ conversion of the starting alkaloid.

(C) Radiolysis of ca $10 \%$ solution of carboline 1a in chlorobenzene in a presence of $3 \%$ of the $\mathrm{N}$-hydroxythiopyridone (2) yielded in $40 \%$ transformation of the alkaloid and in ca $90 \%$ transformation of $\underline{2}$.

The radiolysis reaction product ratio strongly depends on the radiation dose and time, substrate concentration, evaporation of solvent during radiolysis, aerobic or anaerobic conditions, etc., and usually displays a considerable variation from one experiment to the other.

\subsection{Oxidation of $\underline{1 a}$ with lead tetraacetate}

This oxidation was modeled under the Finch $[9,12]$ experimental procedures. Yield of crude $\underline{3 a}$ product: $21.2 \%$.

\subsection{Rearrangement of $\underline{3 a}$}

The reaction, carried out by refluxing product $3 \mathrm{a}$ in methylene chloride for 90 min according to the Finch procedures in methanolic sodium methoxide (12) yielded $4 \mathrm{a}(35 \%)$ or in diluted acetic acid yielded compound $\underline{4 b}(29 \%)$, respectively. Both yields are referred to the GC data and the structures were compared to the previuosly synthesised model alkaloids.

\subsection{Deuterated tetrahydro-beta-carbolines}

These products were synthesised using the deuterated reagents or solvents according to the experimental procedures of Gribble [18,19] or ourselves [20] for the carboline synthesis; the $4-\mathrm{d}_{1}(\underline{1 \mathrm{~b}})$, $3,4-\mathrm{d}_{2}(\underline{1 \mathrm{e}})$ and $3,4, \mathrm{~N}_{12}-\mathrm{d}_{3}(\underline{1 \mathrm{~d}})$ isomers were obtained.

\subsection{Reaction of $\underline{1 a}$ with TEMPO or with cumene hydroperoxide}

The equimolar methylene chloride solution of $\underline{1 \mathrm{a}}$ and TEMPO (2,2,6,6-tetramethyl-1-piperdinyloxy radical) or cumene hydroperoxide were stirred at room temperature for $24 \mathrm{~h}$. The excess of reagent was filtred and the solution mixture was washed with water and analysed by the GC-MS. The reaction yields, expressed in starting material conversion for TEMPO was 12 and 23\% for cumene hydroperoxide, respectively.

\subsection{Oxidation of $\underline{1 a}$ or cyclohexene with $\mathrm{Co}(\mathrm{AcAc})_{3}$}

The $10 \%$ solution of $1 \mathrm{a}$ or cyclohexene in toluene was refluxed in presence of catalytic quantities of the cobalt III acetonylacetate for $12 \mathrm{~h}$ under an oxygen flow. The solution was concentrated in vacuo and examined by the GC-MS. The benzyl alkohol yield, as originating from the the solvent (toluene) oxidation and being the major product of this reaction, is omitted from the reaction yield calculation. All oxidation product yields are calculated from the GC results and reported in the text to the substrate conversion.

Cyclohexene oxidation: total conversion $47 \%$; carboline 1a oxidation: total conversion $18 \%$ (products $\underline{7 \mathrm{a}}$ and $\underline{7 \mathrm{f}}$. 


\section{Acknowledgements}

We would like to thank Professor D.R.H. Barton for constant inspiration, the New Brunswick Health Department and the Faculty of Research and Graduates Studies of the Universite de Moncton for financial support of this work. This project was realised with the unestimable assistance of Ms Suzy Robine and the Centre d'Études Nucléaires of Saclay (Nuclear Research Center of Saclay, France) and the logistic help of the Université Cergy-Pontoise (professor S. Jugé laboratory).

\section{References}

[1] D.H.R. Barton, J. Boivin, K. Schwartzentruber, N. Ozbalik, D. Gaudin and K. Jankowski, Spectroscopy Letters 20 (1987), 963.

[2] De Wei Lu and K. Jankowski, Spectroscopy 11 (1993), 59.

[3] F.A. Fares and K. Jankowski, Heterocycles 34 (1992), 2109.

[4] J. Boivin, D. Gaudin, D. Labrecque and K. Jankowski, Tetrahedron Letters 31 (1990), 2281.

[5] M. Delaforge, M. Jaouen, H. Virelizier and K. Jankowski, Bioorg. Med. Chem. (submitted, 1996).

[6] K. Jankowski, M. Delaforge, J.-P. Girault and J. Gharbi, Bioorg. Med. Chem. 3 (1995), 587.

[7] D.H.R. Barton and W.D. Ollis, Comprehensive Organic Chemistry, Vol. 4, P.G. Sammes, ed., Pergamon Press, New York, 1986, p. 442.

[8] J. Boivin, E. Crepon and S.Z. Zard, Bull. Soc. Chim. Fr. 129 (1992), 145.

[9] D.H.R. Barton, D. Crich and D. Motherwell, J. Chem. Soc. Chem. Comm. (1983), 993; Tetrahedron 41 (1985), 3901.

[10] N. Finch, G.W. Gemeden, I.H. Hsu and W.I. Taylor, J. Amer. Chem. Soc. 85 (1963), 1520.

[11] N. Finch and W.I. Taylor, J. Amer. Chem. Soc. 84 (1962), 3871.

[12] A.J. Swallow, in: MTP International Review of Science. Free Radical Reactions, D.H. Hey and W.A. Waters, eds, Butterworths, London, 1963, p. 263.

[13] K. Gricebaum, Angew. Chem. Int. Engl. Ed. 9 (1970), 373.

[14] J.K. Kochi, Free Radicals, Wiley, New York, 1973, Chapter 23.

[15] A.L.J. Beckwith and S.J. Brumby, J. Chem. Soc. Perkin. Trans. I (1987), 1801.

[16] N. Finch, W.I. Taylor and P.R. Ulshafer, Experientia 19 (1963), 296.

[17] N. Ami, E. Yamanaka, J. Endo, S. Sakaiand and J. Haginiwa, Tetrahedron 29 (1973), 2015 (also see references in Barton [7]).

[18] G.W. Gribble, J. Org. Chem. 37 (1972), 1833

[19] G.W. Gribble, P.D. Lord, J. Skotnicki, S. Dietz, J.T. Eaton and J.L. Johnson, J. Amer. Chem. Soc. 96 (1974), 7812.

[20] K. Jankowski, S. Godin and N.E. Cundasawmy, Can. J. Chem. 52 (1974), 2064.

[21] E.G. Rosantsev and V.D. Sholle, Synthesis 190 (1971), 401.

[22] A. Cabrera, A.M. Perez, M. Salmon and N. Rosas, Synth. React. Inorg. Met.-Org. Chem. 25 (1995), 1507.

[23] H. Alper and M. Harustiak, J. Mol. Catal. 84 (1993), 87. 


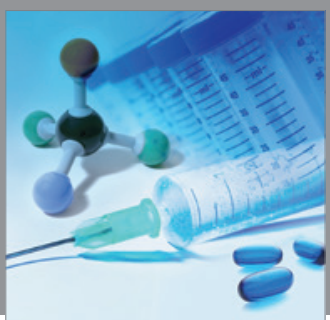

International Journal of

Medicinal Chemistry

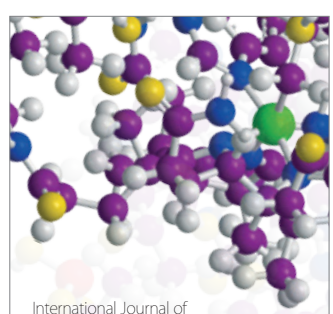

Carbohydrate Chemistry

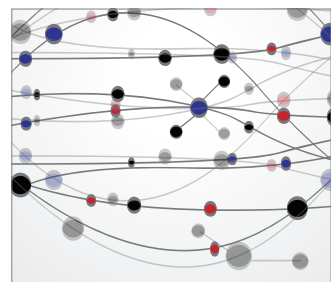

The Scientific World Journal
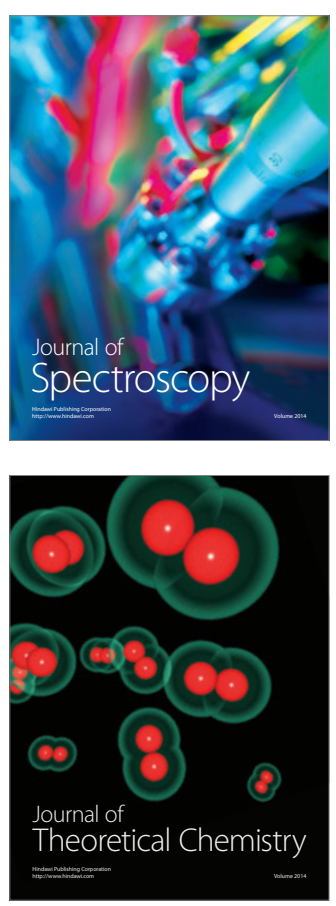
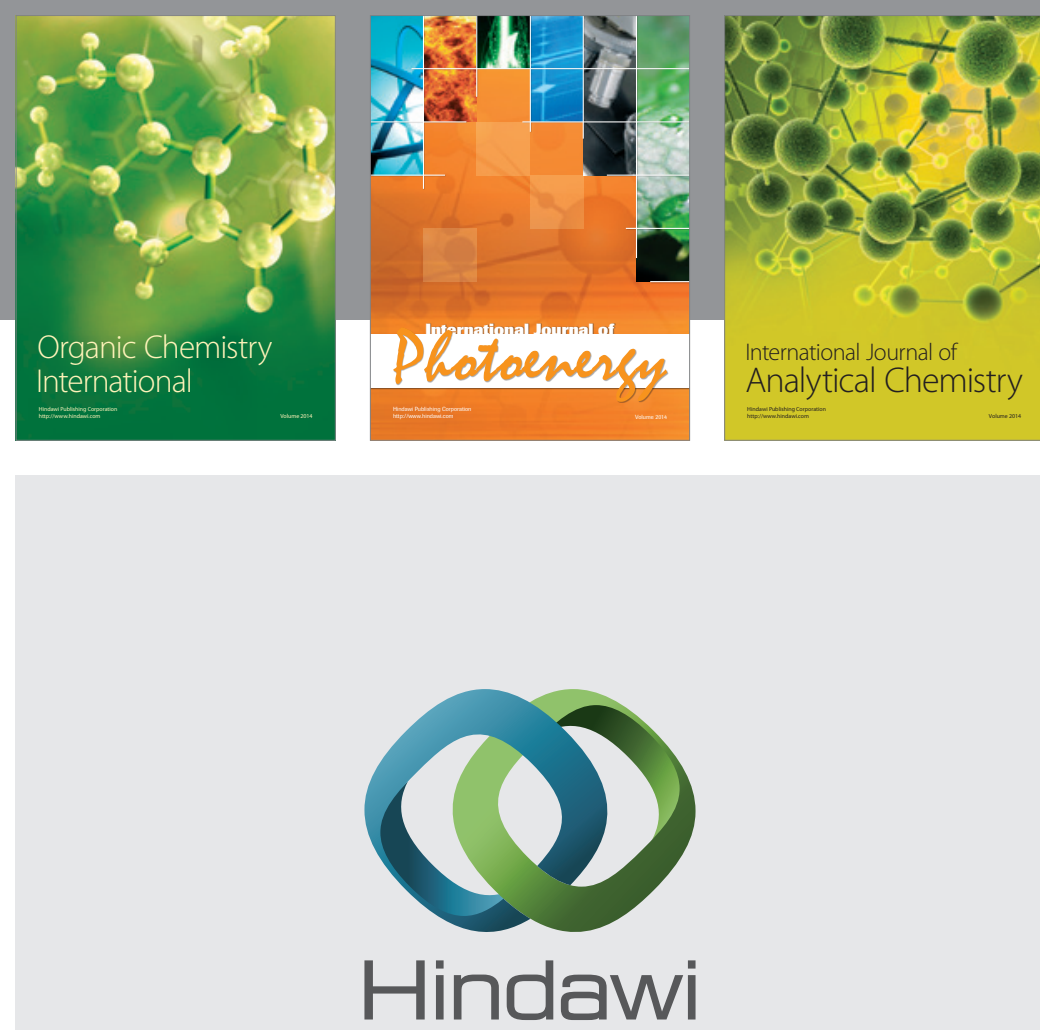

Submit your manuscripts at

http://www.hindawi.com
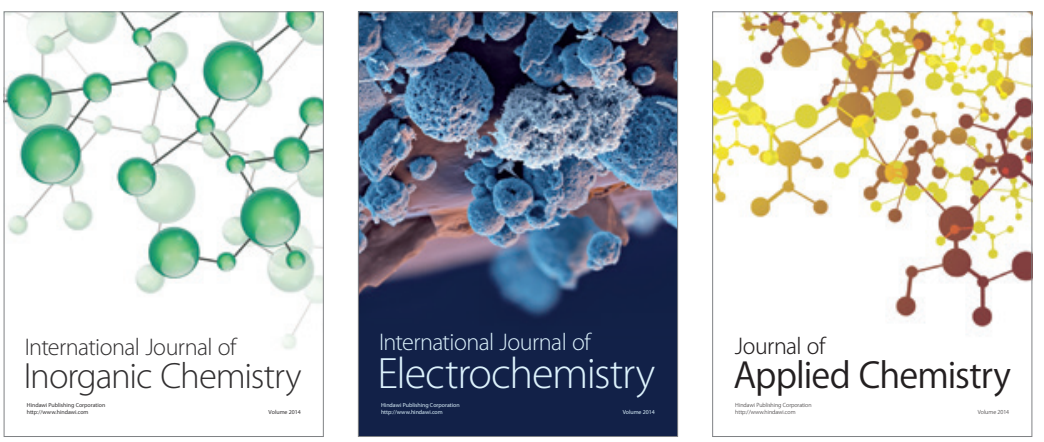

Journal of

Applied Chemistry
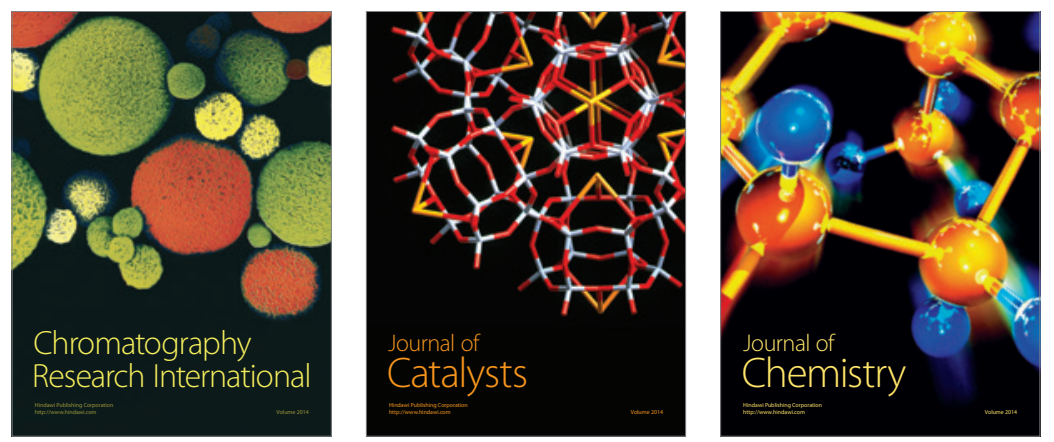
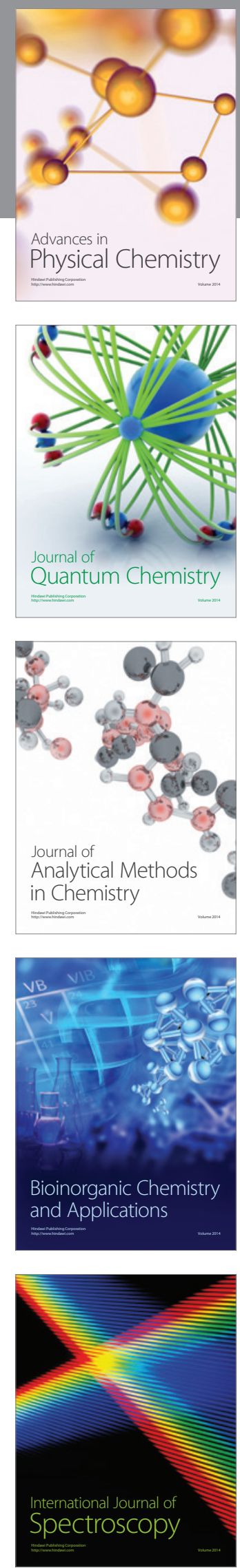